Journal of the Electrochemical Society, Vol. 143, No. 6, 1996, pp.1834-1840.

ISSN: (Print 0013-4651) (Online 1945-7111)

DOI: $10.1149 / 1.1836912$

http://www.electrochem.org/

http://scitation.aip.org/JES

http://scitation.aip.org/getpdf/servlet/GetPDFServlet?filetype=pdf\&id=JESOAN000143000006001834000001\&idty pe $=$ cvips \&prog $=$ normal

(C) The Electrochemical Society, Inc.1996. All rights reserved. Except as provided under U.S. copyright law, this work may not be reproduced, resold, distributed, or modified without the express permission of The Electrochemical Society (ECS). The archival version of this work was published in Journal of the Electrochemical Society, Vol. 143, No. 6, 1996, pp.1834-1840.

\title{
Repassivation of Pits in Aluminum Thin Films
}

\section{G. S. Frankel, ${ }^{\mathrm{a}, \mathrm{b}}$ J. R. Scully, ${ }^{\mathrm{c}}$ and C. V. Jahnes ${ }^{\mathrm{a}}$}

${ }^{a}$ IBM Research Division, T. J. Watson Research Center, Yorktown Heights, New York

${ }^{\mathrm{b}}$ Present address: Department of Materials Science and Engineering, The Ohio State University, Columbus, OH.

${ }^{c}$ Department of Materials Science, University of Virginia, Charlottesville, Virginia

\begin{abstract}
The effect of metal film thickness on repassivation of pits in sputter-deposited Al thin films was investigated in chloride solutions. The repassivation potential and the critical current density, which is the pit current density below which pits stop growing, were determined for pits in Al thin films ranging from $100 \AA \hat{\AA}$ to $43 \mu \mathrm{m}$ in thickness. The repassivation potential first decreased as thickness increased from 100 to $4350 \AA$, and then increased as the film thickness increased further. This behavior was found to be a consequence of the pit currentdensity/potential relationship. The critical current density, a more informative parameter, decreased for increasing metal film thickness, even when the repassivation potential increased. The critical current density is the minimum current density needed to maintain the critical pit environment and prevent repassivation. The repassivation potential for a given metal film thickness is the potential at which the pit current density drops below the critical value. Masstransport and ohmic resistance both increase as the metal film thickness increases, but the former enhances pit stability and the latter destabilizes pitting in this system. Pit repassivation, and thus stability, are strongly influenced by mass-transport considerations for pits in very thin pits, even though dissolution at low potentials is not under pure mass-transport control. Ohmic effects become increasingly important as the film thickness increases.
\end{abstract}

\section{Introduction}

It is widely accepted that there are critical potentials associated with pitting corrosion. ${ }^{1,2}$ The pitting potential or nucleation potential, and the repassivation or protection potential are often determined by cyclic polarization measurements. The pitting potential, $E_{\mathrm{P}}$, the potential at which the current starts to increase rapidly above the background passive current density, is generally considered to be the value above which pits will nucleate. The repassivation potential, $E_{\mathrm{R}}$, which has been defined by various characteristic points along the reverse-scan portion of the cyclic polarization curve, is generally considered to be the value at which growing pits repassivate, or the value above which pits can grow.

This view of pitting corrosion is useful for comparing the susceptibility of various alloys in a given environment or the aggressiveness of various environments toward a given material. However, there is considerable experimental evidence suggesting that this simplistic interpretation does not adequately describe the mechanism of pitting corrosion. First, the potentiodynamically determined pitting potential of most materials often exhibits a wide scatter, of the order of hundreds of millivolts. Furthermore, $E_{\mathrm{P}}$ is in many cases a function of experimental parameters such as potential scan rate. The existence of metastable pits, pits that initiate and grow for a period at potentials well below the pitting potential, ${ }^{3}$ provides clear 
evidence in contradiction to the previously mentioned definition of the pitting potential. The meaning of the repassivation potential has also been called into question. Wilde showed that $E_{\mathrm{R}}$ of ferritic stainless steel decreased (became more negative) with increasing values of the current density at which the potential scan direction was reversed. ${ }^{4,5}$ So deeper pits apparently repassivated at lower potentials. In contrast, the repassivation potential for pits in $\mathrm{Al}$ seemed to be relatively independent of the extent of prior pit growth for a limited number of experiments. ${ }^{6}$ Some have found a similar lack of dependence of $E_{\mathrm{R}}$ on prior growth for pits in stainless steel, but only after the passage of large charge densities. ${ }^{7}$

Thompson and Syrett have suggested that the highest observed repassivation potential is the critical potential in pitting. ${ }^{8}$ They indicated that this value is associated with repassivation of very small pits, and is close to the lowest observed value of pitting potential. No means was suggested, however, for accurate and reproducible determination of this critical potential.

In fact, determination of repassivation potentials from cyclic potentiodynamic polarization curves is often unclear, and various characteristic values of the reverse scan have been used; the corrosion potential, the intersection with the upward scan, and the inflection point at which the current again becomes potential-independent. ${ }^{6}$ The confusion partially stems from the fact that different types of current hystereses are observed for passivating metals. In many materials the passivation current density at a given potential during the reverse scan is lower than during the upward scan because of thickening of the passive film at more noble potentials. In contrast, the passive current density of $\mathrm{Al}$ is often higher during the downward scan after some pit growth at high potentials. ${ }^{9}$ Furthermore, interpretation of the current flowing from an electrode that may contain many pits of varying size is fraught with difficulty as the individual pits of varying size may repassivate at different potentials. Other methods have been used for determination of pitting and repassivation potentials, ${ }^{1}$ but they involve similar uncertainties.

The cessation of pit growth during reverse-potential scanning has been attributed to either deactivation or repassivation. ${ }^{10}$ Deactivation refers to a lowering of the true potential at the pit surface (after accounting for the potential drop down the pit) to a value below the corrosion potential of the active metal in the aggressive pit solution. Repassivation involves a more complex view of the pitting phenomenon. Cation hydrolysis often causes the pit solution $\mathrm{pH}$ to decrease and to be strongly dependent on the pit current density and mass-transfer characteristics of the pit. Chloride ions migrate into the pit from the bulk, increasing the chloride concentration in the pit. As the pit bottom potential decreases during the reverse scan, the pit current density decreases, and the pit solution becomes less concentrated and less acidic. At some point the combination of a more negative pit bottom potential and a less concentrated pit solution results in cessation of pit growth by repassivation of the pit surface. Since freshly repassivated pits usually do not reactivate upon a subsequent small increase in potential above $E_{\mathrm{R}}$, the concept of repassivation is a more likely scenario.

Clear determination of the pit growth cessation mechanism is complicated by problems associated with accurate measurement of the pit current density and its potential dependence. In order to assess the current density for a pit in a bulk sample, the current coming from a single pit and the pit surface area are required. In certain cases it has been possible to form a single pit. ${ }^{3,11-}$ ${ }^{15}$ However, an assumption regarding pit geometry (typically that it is hemispherical) is needed to transform current into current density. For pits in Al, the current associated with hydrogen evolution can be a significant fraction of the total anodic current, thus reducing the measured current. ${ }^{16}$ Experiments with artificial or lead-in-pencil pits have been successful in providing information on pit current density and stability. ${ }^{17-19}$ This arrangement cannot be used, however, 
to study the behavior of very small pits. The current-potential relation in pits is further complicated by the nonsteady-state nature of pit growth; the current density of pits typically decreases with time, (although sub-micron-sized metastable pits in stainless steel may grow with a constant current density. $)^{3}$

Some of the problems associated with studying pits in bulk samples are mitigated by monitoring the growth of pits in thin metallic films on inert substrates with video imaging equipment. ${ }^{16,20,21}$ Pits quickly perforate metallic films with thickness on the order of thousands of angstroms, reach the inert substrate, and then grow two-dimensionally with vertical sidewalls. Analysis of images of growing pits in thin films allows accurate determination of the anodic pit current density because the entire pit is visible during growth; no assumptions regarding pit geometry are required. At a given potential, pits in thin metallic films grow at a constant current density because there is no increasing dimension to result in an increasing ohmic path or diffusion length. For the same reason, the repassivation potential for pits in thin films is reproducible.

Pits in thin films have been considered two-dimensional steady-state analogs to small pits in bulk samples. ${ }^{16,20,21}$ Pits in thin films imitate, to a certain extent, small pits in threedimensional samples because of the small pit depth, which is limited to the metal film thickness once the pit perforates the metal film. Unlike the behavior of ever deepening pits in bulk samples, the constant pit depth of pits in thin films provides a steady-state situation, thus allowing extended periods of time for studying pits of a given depth. By studying pits in films of varying thickness, it is possible to infer the behavior of a small pit in a bulk sample as it grows.

The effect of metal film thickness on the behavior of pits in $\mathrm{Ni}-20 \% \mathrm{Fe}$ was previously studied. ${ }^{20}$ The current-density/potential relationship was found to vary with thickness. The limiting current density at high potentials and the slope $d i / d E$ at lower potentials both decreased as the thickness increased. It was shown that this behavior resulted from increasing diffusional and ohmic resistances, respectively, for thicker films. Remnant passive film, resulting from dissolution of the underlying metal, was found to overhang the active pit wall and influence mass transport. The repassivation potential for pits in NiFe films decreased with increasing film thickness, similar to the behavior mentioned above for pits of increasing depth in ferritic stainless steel. ${ }^{4,5}$

A more recent work focused on $E_{\mathrm{R}}$ and the critical current density, $i_{\text {crit }}$, for sputtered $\mathrm{Al}$ alloy thin films. ${ }^{21} i_{\text {crit }}$ is the current density below which a pit repassivates, or the lowest current density at which a pit can grow. Because of the nature of pits in thin film samples, $E_{\mathrm{R}}$ and $i_{\text {crit }}$ are extremely reproducible. Alloying $\mathrm{Al}$ with any of a number of elements increased $E_{\mathrm{R}}$ substantially, by as much as $1.5 \mathrm{~V}$ in some cases, while having little effect on $i_{\text {crit }}$. This indicated that alloying improved pitting resistance primarily through a reduction in the ability of pits to maintain the critical local environment, as opposed to altering the severity of the critical local environment. The notion of a critical current density for pits in thin films is closely related to the work of Galvele who found that the product of pit depth, $x$, and current density, $i$, determines the extent of acidification in a pit. ${ }^{22}$ Critical values of $x \cdot i$ were associated with passivation. For pits in thin film samples, which have a constant pit depth, this product simply reduces to a critical current density. The critical local environment corresponding to this critical current density is not well known for the case of Al. Using lead-in-pencil electrodes, the critical environment has been determined for stainless steel, $\mathrm{Fe}-\mathrm{Cr}$, and $\mathrm{Ni}$ alloy 600 to be 20 to $60 \%$ of the saturation concentration. ${ }^{23,24}$ Newman has assumed that the critical concentration for $\mathrm{Al}$ is $2 M$, about $65 \%$ of the saturation concentration. ${ }^{25}$ 
Very recently, a study of pitting in Al thin films with thickness ranging from 500 Á to 5 $\mu \mathrm{m}$ was reported. ${ }^{26}$ No difference in the pitting potential, as determined by potentiodynamic polarization in $0.04 \mathrm{M} \mathrm{Cl}^{-}$solutions, was observed.

In the present study, the effects of metal film thickness on the $E_{\mathrm{R}}$ and $i_{\text {crit }}$ for pits in $\mathrm{Al}$ films are described. As with bulk samples, pits in Al thin films behave differently from pits in nickel and ferrous alloys because of the copious evolution of hydrogen gas at the pit surface during growth. The motion of gas bubbles enhances mass transfer, and also lifts the undercut passive film away from the active pit wall to some extent. The repassivation potential and critical current density of pits in Al films with a range of thickness is reported here. The thickness dependence of repassivation potential, upon making the analogy to deepening pits in bulk samples, provides evidence for the pit stability criteria during pit growth in Al.

\section{Experimental}

Al films with thickness ranging from $100 \AA \AA$ to $43 \mu \mathrm{m}$ were deposited by dc magnetron sputtering a $99.999 \%$ pure Al target using a rotating-platform, load lock deposition tool with a base pressure of about $2 \times 10^{-7}$ Torr. With the platform rotating, the samples moved into and out of the deposition zone at $5 \mathrm{rpm}$, yielding a nominal Al deposition rate of $250 \AA \AA / \mathrm{min}$. In the deposition zone the instantaneous rate was about $2500 \AA$ Á/min. The substrates were either quartz, or $\mathrm{Si}$ covered with $\mathrm{Si}_{3} \mathrm{~N}_{4}$ or $\mathrm{SiO}_{2}$. Samples were pressed against the bottom of a Plexiglas electrochemical cell, facing upward, using a Teflon o-ring that exposed an area of about 0.78 $\mathrm{cm}^{2}$. The cell also contained a Pt counterelectrode, an SCE reference electrode, and a sleeve allowing insertion of ah optical probe for recording images of the growing pits. ${ }^{16}$ The electrolyte was stagnant, and exposed to air.

In most experiments, pits were initiated by stepping the potential to a high value, between -0.5 and $0 \mathrm{~V}$ SCE. Within seconds, the potential was then decreased to a value near the repassivation potential. The potential was held at a given value for a period of 15 to $120 \mathrm{~s}$, and then further decreased, typically by $10 \mathrm{mV}$, until the pit repassivated. The period of time at each potential depended inversely on the pit perimeter velocity. A longer time was needed at a given potential if the conditions were such that the pit perimeter moved slowly during pit growth. As discussed in a previous work, ${ }^{21}$ observation of the growing pit enabled accurate determination of the repassivation potential. Growth of pits in these Al thin films was accompanied by the emission of a steady stream of hydrogen bubbles; pit repassivation was also clearly connected with the cessation of hydrogen bubble formation.

The anodic pit current density for pits grown in $1.0 \mathrm{M} \mathrm{NaCl}$ was determined at each applied potential by analysis of the pit images using an approach described previously ${ }^{16,20}$ The average anodic pit current density at any given time is proportional to the derivative of the pit bottom area with respect to time divided by the pit perimeter at that time. Several pits typically formed, so the measured current was not useful, and the current density was determined solely by image analysis. The $1.0 \mathrm{M} \mathrm{NaCl}$ electrolyte was used for the current density analysis because the morphology of pits in thin Al films becomes extremely convoluted near the repassivation potential in dilute chloride solutions, and the determination of current density becomes extremely difficult. ${ }^{16}$ This analysis was further limited to pits in the thinnest Al films owing to the large accumulation of hydrogen bubbles at the perimeter of pits in thick films. Hydrogen was evolved in pits of all thicknesses. However, pits in thicker films generate more total hydrogen gas because of the larger active pit area (thus larger hydrogen evolution current), and these bubbles can accumulate and obscure the pit perimeter. The anodic pit current density for pits in films 
with thickness of 1430 to $9670 \AA$ Á was calculated by a simplified technique. Hydrogen bubbles tended to accumulate only at certain locations, so local regions of the pit perimeters not obscured by bubbles could be analyzed. The local anodic current density is proportional to the velocity of the pit perimeter, which was determined by the distance moved by the perimeter segment divided by the time. Note that the standard approach determines the average pit perimeter velocity over the whole perimeter. Measurements of pit current density from the velocity of local pit perimeter regions were found to be accurate to about \pm 5 to $10 \%$. This was determined by analyzing pits in thin films for which the pit current density could also be measured by the standard approach. Pits in 6.7 and $43 \mu \mathrm{m}$ thick films were totally obscured by bubbles, so that even the simplified analysis was impossible. However, it was still possible to determine the repassivation potential for pits in these thick films by visual observation. Pit growth was always accompanied by generation of new hydrogen bubbles, or growth of existing bubbles. When the pits stopped growing, the hydrogen bubbles actually decreased in size slowly with time as the hydrogen gas slowly dissolved into the electrolyte. So $E_{\mathrm{R}}$ for the thickest films was determined to be the potential at which the bubble of hydrogen gas on top of the pit stopped increasing in size and began decreasing.

Some of the pits grew into the crevice region during the experiment because they initiated at the edge of the exposed area. However, image analysis was performed only on pits away from the crevice, or on pits near the edge before they intersected the crevice.

\section{Results}

The average pit current densities, determined by image analysis, for pits in replicate $\mathrm{Al}$ films of various thickness are given in Fig. 1 as a function of pit growth potential in $1.0 \mathrm{M} \mathrm{NaCl}$. As was observed previously for pits in thin films, ${ }^{16,20,21}$ the pit current density varied little with time at a given potential, and the average value over the time of growth at each potential is given in Fig. 1. The $i$ - $E$ curves for pits in thin films typically exhibit a limiting current region at high potentials where the pit current density is relatively independent of growth potential, and a

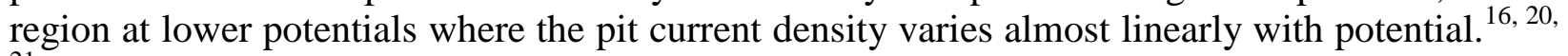

21 Since the objective of the experiments in this work was to determine the repassivation potential and critical current density, only potentials near the repassivation potential were investigated. Therefore, the high potential limiting-current regions of $i$ - $E$ curves are not found in Fig. 1 and the pit current density is approximately linearly dependent on potential. For each experiment, the pit repassivated when the potential was decreased by a further $10 \mathrm{mV}$ step below the point representing the lowest potential and current density. The potential at which the pit stopped growing was determined to be the $E_{\mathrm{R}}$, and the lowest current density at which the pit could grow (at the potential that was $10 \mathrm{mV}$ above the $E_{\mathrm{R}}$ ) was defined as $i_{\text {crit }}$. While slightly different values of $E_{\mathrm{R}}$ and $i_{\text {crit }}$ might be found between the last two values of applied potential, the $10 \mathrm{mV}$ resolution was deemed to be sufficient.

As was observed for $\mathrm{NiFe},{ }^{20}$ the slope $d i / d E$ decreases as the thickness increases, although the thinnest samples have similar slopes. The $i$ - $E$ curves for the 150 and $100 \AA$ thick films are offset to more noble potentials compared to the thicker films. This phenomenon was not observed for NiFe, but films thinner than 300 A were not examined in that work. ${ }^{20}$ 


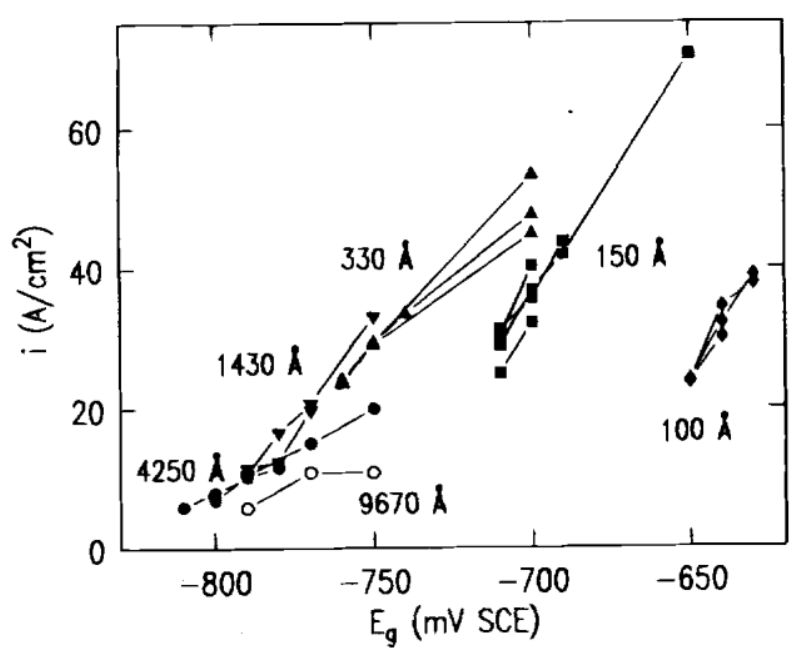

Fig. 1. Average anodic pit current density as a function of pit growth potential for Al thin films of various thickness in 1.0 M NaCl. See text for description of how the repassivation potential and critical pit current density are determined from these data.

The values of $E_{\mathrm{R}}$ and $i_{\text {crit }}$ are seen in Fig. 1 to be extremely reproducible. Figure 2 shows the dependence of $E_{\mathrm{R}}$ on film thickness for two chloride concentrations. The values of $E_{\mathrm{R}}$ in 0.1 $\mathrm{M} \mathrm{NaCl}$ are $80 \mathrm{mV}$ more noble than those in $1.0 \mathrm{M} \mathrm{NaCl}$ at almost every thickness. In both solutions, $E_{\mathrm{R}}$ decreases by $150 \mathrm{mV}$ as the sample thickness increases from 100 to $4250 \AA$. Further increases in thickness result in increases in $E_{\mathrm{R}}$, with 6 and $43 \mu \mathrm{m}$ thick samples exhibiting the same values.

The effect of film thickness on $i_{\text {crit }}$ is summarized in Fig. 3. As described above, $i_{\text {crit }}$ could only be determined for samples in the more concentrated chloride solution, and for films with thickness of $1 \mu \mathrm{m}$ and less. Except for the thinnest sample, $i_{\text {crit }}$, decreases with increasing sample thickness.

\section{Discussion}

The emphasis of this work was on repassivation since the factors $E_{\mathrm{R}}$ and $i_{\text {crit }}$ provide information on the stability conditions for pits in thin films. $E_{\mathrm{R}}$ is the potential at which a pit in a thin film stops growing, and $i_{\text {crlt }}$ is the pit current density below which the pit will stop growing, i.e., the lowest current density at which pit growth can be maintained. These factors are extremely reproducible for pits in thin films, and are independent of pit diameter. If a correlation of the behavior of pits in thin films to that of very small pits in bulk materials is valid, understanding the stability criteria for pits in thin films can help provide information on pit initiation in general. The dependence of $E_{\mathrm{R}}$ and $i_{\text {crit }}$ on film thickness needs to be understood. Before addressing that, however, other aspects of the $i$ - $E$ curves are discussed. 


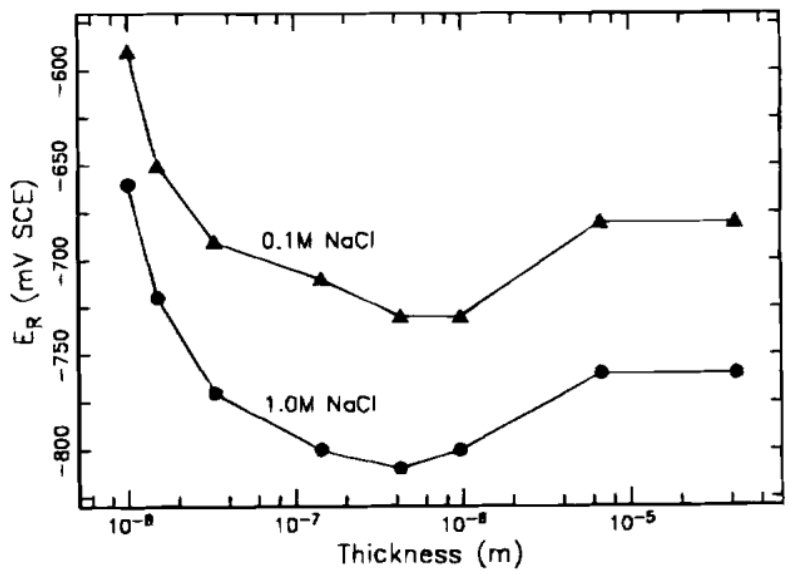

Fig. 2. Repassivation potential as a function of film thickness for $\mathrm{Al}$ thin films in two different $\mathrm{NaCl}$ solutions. Note that the reproducibility of the data points is $\pm 10 \mathrm{mV}$.

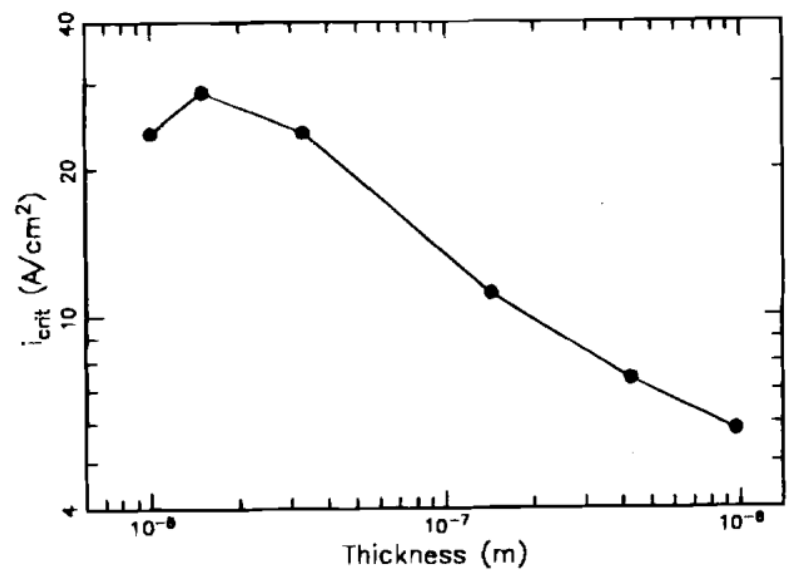

Fig. 3. Critical current density as a function of film thickness for $\mathrm{Al}$ thin films in $1.0 \mathrm{M} \mathrm{NaCl}$.

In a previous paper, ${ }^{16}$ the resistance of current flow to a 2-D pit was estimated using an equation generated by Pierini and Newman for the primary resistance of a thin conductive ring electrode in an insulating plane. ${ }^{27}$ The product of the resistance and the electrolyte conductivity provides a shape factor, $S$, which is representative of the pit geometry

$$
S=\frac{1}{r_{1}}\left\{0.2313-\frac{1}{2 \pi^{2}} \ln \left[1-\left(\frac{r_{0}}{r_{1}}\right)^{3}\right]\right\}
$$

where $r_{0}$ and $r_{1}$ are the inner and outer ring radii, respectively. The 2-D pit geometry can be related to that of a ring by assuming $r,=r_{0}+x$, where $x$ is the pit depth or metal film thickness. Figure 4 shows how the shape factor changes with pit depth and radius. It is clear that the pit radius has a minor influence on the shape factor; the resultant constant diffusion length and ohmic path lead to the steady-state behavior of pits in thin films as has been discussed above. The slopes of the lines in Fig. 4 are about 0.9 , so $S$ varies almost linearly with metal film thickness. 


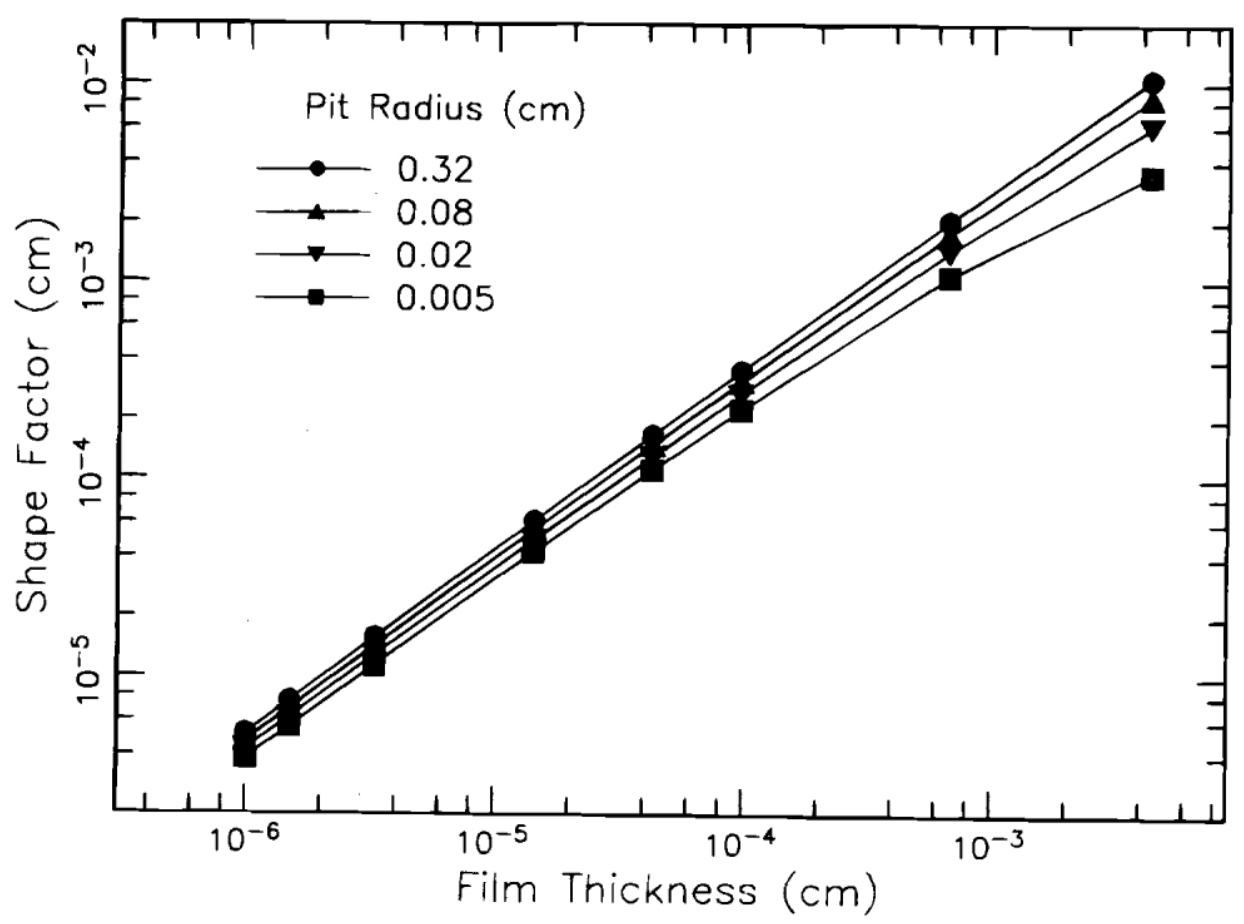

Fig. 4. Shape factor, $S$, as a function of pit depth (or metal film thickness) and pit radius as calculated from Eq. 1.

Assuming no convective effects, $S$ is both the diffusion length and, as mentioned above, the ohmic resistance after dividing by the solution conductivity, $\mathrm{K} \cong 0.1 \Omega^{-1} \mathrm{~cm}^{-1}$ for $1.0 \mathrm{M} \mathrm{NaCl}$, so the inverse slope of the $i-E$ curve for pit growth would be predicted to be $5 \times 10^{-4} \mathrm{ft} \mathrm{cm}^{2}$ for a film thickness of $1430 \AA$. The inverse of the slope in Fig. 1 for this thickness gives a total resistance of $2.3 \times 10^{-3} \mathrm{ft} \mathrm{cm}^{2}$. A potential component calculation for 2-D pits in NiFe determined that the ohmic resistance should account for about one-half of the total observed $d E / d i$ for this thickness. ${ }^{20}$ Activation and concentration overpotentials accounted for the rest. The fact that the predicted ohmic resistance is much less than half of the total observed resistance suggests that the effective solution conductivity is smaller than the bulk conductivity. The presence of hydrogen bubbles at the active surface area apparently decreases the local conductivity considerably.

Hydrogen bubbles can also play a role in altering the diffusion length by enhancing convection. Whereas this study did not focus on the diffusion-limited region of pit growth, it is possible to make use of data reported earlier. The limiting current, $i_{\text {lim }}$, for pits in $1500 \AA$ thick Al films was found to be about $30 \mathrm{~A} / \mathrm{cm}^{2} .{ }^{16}$ Assuming a saturation concentration for $\mathrm{A} 1 \mathrm{C}_{3}$ and diffusivity for $\mathrm{Al}^{3+}$ of $3.11 \mathrm{M}$ and $3 \times 10^{-7} \mathrm{~cm}^{2} / \mathrm{s},{ }^{28}$ respectively, the effective diffusion layer thickness, $\delta=n F D C_{\text {sat }} / i_{\text {lim }}$, may be estimated to be $9.0 \times 10^{-6} \mathrm{~cm}$. This value is somewhat less than the shape factor, or predicted diffusion length, for this geometry of $5 \times 10^{-5} \mathrm{~cm}$. As mentioned, the hydrogen bubbles that act to increase $d E / d i$ by decreasing conductivity can also enhance convection and decrease the diffusion layer thickness. The local cathodic current density attributable to hydrogen evolution for pits in $1500 \AA$ thick films in $0.1 \mathrm{M} \mathrm{NaCl}$ was found to be about $1.5 \mathrm{~A} / \mathrm{cm}^{2} .{ }^{16} \mathrm{Ibl}$ showed that the diffusion layer thickness on planar electrodes decreased by about a factor of ten as the hydrogen current density increased from 0.1 to $1 \mathrm{~A} / \mathrm{cm}^{2} .^{29}$

Buzza and Alkire recently presented an analysis of the transport conditions for small 
(about $10 \mu \mathrm{m}$ diam) 3-D pits in Al by estimating the Peclet number $(\mathrm{Pe}=v l / D$ where $v$ and $l$ are the characteristic velocity and length, respectively), which predicts convective control for Pe $>1$ and diffusive control for $\mathrm{Pe}<1 .{ }^{15}$ They suggested that convection plays a minor role in transport, even in the presence of gas evolution, because Pe was found to be somewhat less than 1 . This finding is in contrast to the evidence in the present study that hydrogen evolution increases mass transport. In their analysis, however, Buzza and Alkire assumed a diffusion coefficient of $1 \times 10^{-}$ ${ }^{5} \mathrm{~cm}^{2} / \mathrm{s}$, since that is a typical value for metal salts. ${ }^{15} \mathrm{D}$ for $\mathrm{Al}^{3+}$ in concentrated chloride solutions was determined by Beck to be about $3 \times 10^{-7} \mathrm{~cm}^{2} / \mathrm{s} .{ }^{28}$ Pe increases by a factor of 30 if this smaller diffusivity is used, suggesting that convection may indeed dominate transport for pits in Al.

Interestingly, the diffusion layer thickness is $6.6 \times 10^{-5} \mathrm{~cm}$ for pits in a NiFe film of similar thickness in $0.1 \mathrm{M} \mathrm{NaCl}$ calculated from the limiting current density of $20 \mathrm{~A} / \mathrm{cm}^{2},{ }^{20}$ and assuming $C_{\text {sat }}=4.55 \mathrm{M}$ for $\mathrm{NiCl}_{2}{ }^{30}$ and $D=1.5 \times 10^{-6} \mathrm{~cm}^{2} / \mathrm{s}$ for $\mathrm{Ni}^{2+}$. ${ }^{31}$ (Note that a further simplification here is the assumption that the $\mathrm{Ni}-20 \mathrm{Fe}$ alloy acts like pure $\mathrm{Ni}$.) This measured value is somewhat larger than the diffusion length predicted from the shape factor, and was attributed in an earlier work to the influence of undermined passive film acting as a barrier to transport. ${ }^{20}$ Hydrogen, which was not evolved at the pit surface of NiFe samples, eliminates the barrier of the undermined passive film by lifting it up and away from the active pit area. In doing so it enhances transport via convection and reduces conductivity by replacing electrolyte volume with resistive gas.

A notable feature of Fig. 1 is that the $i-E$ curves for the 150 and 100 A thick films are offset to higher potentials by about 50 and $100 \mathrm{mV}$, respectively, compared to the thicker films. No such offset was observed for NiFe films of thickness down to $300 \AA .^{20}$ The effect is similar, though smaller in magnitude, to the influence of alloying $\mathrm{Al}$ with small amounts of $\mathrm{Cr}$ or $\mathrm{Nb} .{ }^{21}$ The $i-E$ curves for $\mathrm{Al}-4 \% \mathrm{Nb}$ and $\mathrm{Al}-3 \% \mathrm{Cr}$ were offset to higher potentials from that of pure $\mathrm{Al}$ by about 600 and $300 \mathrm{mV}$, respectively. The cause of the ennoblement of pit growth in 150 and 100 A thick pure Al films compared to thicker Al films may be the same as that suggested for the sputtered Al binary alloys. ${ }^{21}$ Pure metallic Al is extremely active and reactive. Additions of alloying elements can easily increase the reversible potential or decrease the exchange current density for metal dissolution. A simple potential component calculation showed clearly how these factors can cause the $i-E$ curve to shift to higher potentials. ${ }^{20}$ For very thin Al films, it is likely that oxygen is the alloying element causing this ennoblement. Small amounts of oxygen in $\mathrm{Al}$ can have a large influence on the pitting resistance of Al. It has been reported that Al sputterdeposited with a base pressure of $6 \times 10^{-6}$ Torr has a pitting potential $500 \mathrm{mV}$ above that of bulk Al owing to the incorporation of a small amount of oxygen from the sputtering environment. ${ }^{32}$ It is the experience in this laboratory that the pitting behavior of thin film Al is similar to that of bulk Al if the base pressure is about $2 \times 10^{-7}$ Torr or lower and the deposition rate is high. Even with the sputtering conditions used in this work, there is some incorporation of oxygen, both at the interface with the substrate, which is the first part of the film to be deposited, and the top layer that is exposed to air. It has been shown that the initial layers of $\mathrm{Al}$ deposited on $\mathrm{SiO}_{2}$ or $\mathrm{Si}_{3} \mathrm{~N}_{4}$ will reduce the oxide or nitride to form $\mathrm{A} 1_{2} \mathrm{O}_{3}$ or $\mathrm{A} 1 \mathrm{~N}$, respectively ${ }^{33,34}$ Oxygen that was adsorbed onto the surface of a substrate previously exposed to air will also be incorporated. So the $\mathrm{Al}$ closest to the substrate will contain some amount of oxygen and/or nitrogen. Reaction of the top surface upon exposure to the atmosphere to form the surface oxide film also adds oxygen. The amount of oxygen incorporated at the substrate interface is much less than that associated with the surface oxide. As the film thickness decreases, these two regions become an increasing 
portion of the total film thickness. The 100 and $150 \AA$ thick films thus act like Al alloys, and exhibit ennobled $i-E$ curves because of this incorporated oxygen and nitrogen. It must be concluded that the correlation of the behavior of pits in thin films to small pits in bulk Al may only be valid for films thicker than 200 to $300 \AA$ Á.

For each thickness in Fig. 1 the point at the lowest current density and potential represents the critical current density, $i_{\text {crit }}$, and the potential $10 \mathrm{mV}$ above the repassivation potential, $E_{\mathrm{R}}$. The chemical environment at the pit wall will depend on the pit current density, the masstransport conditions, and the equilibrium constants for the reactions that occur in pit environments, such as hydrolysis. This environment is normally dependent on the exact geometry of the pit, which influences the mass transport. As the potential is stepped downward during an experiment and the pit current density decreases, the local cation concentration decreases, the local $\mathrm{pH}$ increases, and the chloride concentration decreases. When the pit current density decreases below $i_{\text {crit, }}$, the local environment becomes sufficiently dilute to allow repassivation of the pit wall, and pit growth ceases. $i_{\text {crit }}$ decreases with increasing film thickness because the rate of mass transfer is reduced and less current density is needed to maintain the critical conditions. The log-log plot of Fig. 3 shows that $i_{\text {crit }}$ varies approximately with thickness, $x$, to the $-1 / 2$ power. A simple diffusion model would predict that the current density needed to maintain a fixed concentration at the pit surface should vary with the inverse of the shape factor given in Eq. 1 and Fig. 4. Therefore, $i_{\text {crit }}$ should vary with $x^{-0.9}$. The difference in dependence on thickness may again result from convection associated with hydrogen evolution so that the diffusion layer does not build up to the extent that it should. Pit growth near the repassivation potential is not under mass-transport control. Nonetheless, the repassivation is determined by the local environment, which is strongly dependent on transport considerations.

The critical concentration for repassivation can be estimated from the critical current density for the $1430 \AA$ film in the $1 \mathrm{M} \mathrm{Cl}^{-}$solution assuming a limiting current density of 26 $\mathrm{A} / \mathrm{cm}^{2}$ (decreased from the value measured in $0.1 M \mathrm{NaCl}$ owing to the lower concentration gradient) and the values of diffusivity and diffusion layer thickness given above. The result is 1.5 $M$, about $48 \%$ of the saturation concentration. This value is less than the critical concentration assumed by Newman. ${ }^{25}$

As shown in Fig. 2, the repassivation potential first decreases then increases with increasing film thickness. Since the scatter in the $E_{\mathrm{R}}$ data is less than $\pm 10 \mathrm{mV}$, this tendency is real even considering possible error associated with the thinnest films. The shape factor would indicate that both the diffusion length and the ohmic resistance are smallest for the thinnest films. Therefore diffusion is enhanced and ohmic potential drops are minimized for the thinnest films. Correspondingly, diffusion rates decrease and ohmic potential drops increase as the pit depth increases. The influence of film thickness on $E_{\mathrm{R}}$ as shown in Fig. 2 is a result of the opposite influences of diffusion and ohmic potential drops on pit stability in this system.

For samples less than $4250 \AA$ thick, $E_{\mathrm{R}}$ decreases with increasing thickness. This is a reflection of the decreasing $i_{\text {crit }}$; the rate of diffusion decreases as the thickness increases so that a lower current density is needed to maintain or achieve the critical environment for a pit to grow. Correspondingly lower potentials are needed to achieve the lower critical current densities. Of course the potential for a given current density will depend on the slope of the $i-E$ curve, which decreases as the thickness increases.

As the thickness increases past $4250 \AA$, $E_{\mathrm{R}}$ increases again. Since the $i$ - $E$ curves and $i_{\text {crit }}$ were not determined for pits in all of the thick films, there is not a complete set of data to support the mechanism behind this increase. It is possible that $i_{\text {crit }}$ begins to increase as film thickness 
increases past $4250 \AA$, and that higher potentials are needed to achieve these higher current densities. The rationale for such behavior, however, is not clear. In fact, $i_{\text {crit }}$ for pits in $9670 \AA$ films is lower that for pits in $4250 \AA$ films, despite the higher value of $E_{\mathrm{R}}$. A more likely scenario is that the critical current density continues to decrease with increasing film thickness, but higher potentials are required to achieve the lower current densities. This happens because the slope of the $i-E$ curve, $d i / d E$, decreases as the thickness increases. Essentially, the increasing ohmic resistance, which destabilizes pits, overcomes the increasing diffusional resistance, which is a stabilizing influence. For pits in $\mathrm{Al}$, ohmic potential drops are destabilizing because they consume overpotential that would otherwise be available at the pit surface to drive a higher current density.

A recent report was made on the dissolution of holes drilled in Ni samples in $1 \mathrm{~N} \mathrm{H}_{2} \mathrm{SO}_{4}$. ${ }^{35} \mathrm{Ni}$ exhibits a large active peak in this highly acidic environment. It was shown that the ohmic potential drop stabilized dissolution in the hole by shifting the local potential from the passive potential at which the sample was being held into the active region. Under these conditions, a concentrated environment in the occluded region (such as that formed when the hole faced upward) was detrimental to stability, and a more dilute environment region (such as that formed when the hole faced downward) enhanced stability because of the effects on ohmic potential drops. $^{35}$ The situation is much different for the case of pitting in a neutral, dilute solution as in this work on Al. Active pit growth is stabilized by the aggressive local environment that forms in the pit. Ohmic potential drops reduce the potential and current density at the pit surface, and thus also reduce the local pit solution concentration. This action facilitates repassivation.

The behavior of pits in thin Al films of a range of thickness thus sheds light on the critical parameters for pit stability. Ultimately, mass transport determines the stability of pits because the local environment controls passivation. Pit growth is not under total mass-transport control, however, because the critical concentration to prevent repassivation is only about $50 \%$ of the saturation concentration. As has been described previously, pit growth in thin films at potentials below the range of limiting pit current densities is controlled by a combination of ohmic, chargetransfer, and concentration overpotential factors. ${ }^{20}$ Ohmic considerations were found to dominate only for film thicknesses above around 2000 A. The potential required to maintain the critical local environment is thus a direct function of the pit current-density/potential relationship for a pit of a given thickness. As the metal film thickness increases (corresponding to 3-D pits of increasing size), both the mass-transport and ohmic resistances increase. The influences of these resistances on pit growth are opposite in nature, however. As a pit starts to grow, the repassivation potential is initially determined by mass-transport effects. Then as it deepens, ohmic effects dominate.

Buzza and Alkire recently described the repassivation of pits in bulk Al. ${ }^{15}$ Single pits were initiated by laser irradiation in $1.0 \mathrm{M} \mathrm{Cl}^{-}$solution, and grown for a period at $-650 \mathrm{mV} \mathrm{SCE}$. The potential was then stepped downward and the repassivation potential was determined as a function of the amount of charge passed before stepping. $E_{\mathrm{R}}$ decreased from about -770 to -800 $\mathrm{mV}$ as the total charge increased from about 50 to $240 \mu \mathrm{C}$. This charge corresponds to pit radii of 9 to $15 \mu \mathrm{m}$. For larger pits, $E_{\mathrm{R}}$ was $-800 \mathrm{mV}$, independent of pit size. These data are qualitatively similar to the data presented in Fig. 2, though offset to larger dimensions. It is possible to probe the behavior of much smaller pits using thin film samples.

The potential values in Fig. 2 may be considered to be critical potentials of the sort described by Thompson and Syrett. ${ }^{8}$ A pit of a given size will only grow in a stable fashion if the potential is above the $E_{\mathrm{R}}$ for a pit of that size, because only then is the pit current density 
sufficiently large to maintain the critical concentration for a pit of that size. The lowest potential for pit initiation may be the critical potential corresponding to the size of the initial defect. As discussed previously, the measured pitting potential is often close to the repassivation potential, but can be much higher. ${ }^{21}$ For example, an Al thin film aged by exposure to laboratory air for 4 years exhibited a pitting potential that had increased by $200 \mathrm{mV} \cdot{ }^{21}$ However, the pitting potential of a freshly deposited thin film (if the film is deposited with a low base pressure) is similar to that for a freshly prepared bulk sample, and close to the values of repassivation potential shown in Fig. $2 .{ }^{16}$ This variability in pitting potential limits its usefulness as a design or predictive parameter. The repassivation potentials and critical current densities for thin film samples are, in contrast, very reproducible and therefore provide insight into the pitting process.

\section{Conclusions}

The behavior of pits in Al thin films of a range of thickness was investigated, with a focus on the potential range near the repassivation potential. The repassivation potential was found to vary with metal film thickness, decreasing as thickness increased from $100 \AA$ to around $4000 \AA$, and then increasing for thicker films. Pit current density was determined for pits grown in $1.0 \mathrm{M} \mathrm{NaCl}$ by analysis of images of growing pits. The critical current density, the lowest current density at which pits could grow, decreased as thickness increased throughout the range where this measurement was possible. The critical current density is associated with the maintenance of a critical pit environment, whereas the repassivation potential is a consequence of the pit current density/potential relationship. Pit repassivation, and thus stability, are controlled by mass-transport considerations for pits in very thin pits. The repassivation potential is determined by ohmic effects as the film thickness gets large.

\section{Acknowledgments}

The authors appreciate helpful conversations with R. C. Newman, H. S. Isaacs, and C. Pistorius. J.R.S. was supported by the NASA-Langley Research Center La² ST Program and the NSF under DMR-9357463.

IBM T. J. Watson Research Center assisted in meeting the publication costs of this article.

\section{REFERENCES}

1. Z. Szklarska-Smialowska, Pitting Corrosion of Metals, NACE, Houston, TX (1986).

2. M. Pourbaix, L. Klimzack-Mathieu, C. Mertens, J. Meunier, C. Vanleugenhaghe, L. de Munck, J. Laureys, L. Nellemans, and M. Warzee, Corros. Sci., 3, 239 (1963).

3. G. S. Frankel, L. Stockert, F Hunkeler, and H. Boehni, Corrosion, 43, 429 (1987).

4. B. E. Wilde and E. Williams, Electrochim. Acta, 16, 1971 (1971).

5. B. E. Wilde, in Localized Corrosion NACE-3, R. W Staehle, B. F Brown, J. Kruger, and A. Agrawal, Editors, NACE, Houston, TX (1974).

6. M. Yasuda, F. Weinberg, and D. Tromans, This Journal, 137, 3708 (1990).

7. N. Sridhar and G. A. Cragnolino, Corrosion, 49, 885 (1993).

8. N. G. Thompson and B. C. Syrett, ibid., 48, 649 (1992).

9. S. T Pride, J. R. Scully, and J. L. Hudson, This Journal, 141, 3028 (1994).

10. K. K. Starr, E. D. Verink, Jr., and M. Pourbaix, Corrosion, 32, 47 (1976).

11. H.-H. Strehblow and J. Wenners, Z. Phys. Chem. N. F, 98, 199 (1975).

12. R. C. Newman and E. M. Franz, Corrosion, 40, 325 (1984).

13. R. C. Alkire and K. P. Wong, Corros. Sci., 28, 411 (1988).

14. P. C. Pistorius and G. T Burstein, ibid., 33, 1885 (1992).

15. D. W Buzza and R. C. Alkire, This Journal, 142, 1104 (1995).

16. G. S. Frankel, Corros. Sci., 30, 1203 (1990).

17. J. W Tester and H. S. Isaacs, This Journal, 122, 1438 (1975).

18. R. C. Newman, Corros. Sci., 25, 341 (1985). 
19. U. Steinsmo and H. S. Isaacs, This Journal, 140, 643 (1993).

20. G. S. Frankel, J. O. Dukovic, B. M. Rush, V. Brusic, and C. V. Jahnes, ibid., 139, 2196 (1992).

21. G. S. Frankel, R. C. Newman, C. V. Jahnes, and M. A. Russak, ibid., 140, 2192 (1993).

22. J. R. Galvele, ibid., 123, 464 (1976).

23. G. T Gaudet, W T Mo, T. A. Hatton, J. W Tester, J. Tilly, H. S. Isaacs, and R. C. Newman, AlChE J., 32, 949 (1986).

24. U. Steinsmo and H. S. Isaacs, This Journal, 140, 643 (1993).

25. R. C. Newman, Corros. Sci., 37, 527 (1995).

26. S. E. Hernandez, A. J. Griffin, Jr., F. R. Brotzen, and C. F. Dunn, This Journal, 142, 1215 (1995).

27. P. Pierini and J. Newman, ibid., 125, 79 (1978).

28. T. R. Beck, Electrochim. Acta, 29, 485 (1984).

29. N. Ibl, Chem. Ing. Tech., 43, 202 (1971).

30. F Hunkeler and H. Boehni, in Corrosion Chemistry in Pits, Crevices, and Cracks, A.Turnbull, Editor, HMSO, London (1987).

31. R. H. Stokes, S. Phang, and R. Mills, J. Sol. Chem., 8, 489 (1979).

32. R. B. Inturi and Z. Szklarska-Smialowska, Corros. Sci., 34, 1201 (1993).

33. Y. E. Strausser, E. J. Scheibner, and J. S. Johannessen, Thin Solid Films, 52, 203 (1978).

34. J. Avila and J. L. Sacedon, Appl. Phys. Lett, 66, 757 (1995).

35. M. Wang, H. W. Pickering, and Y. Xu, This Journal, 142, 2986 (1995). 